\title{
A mighty mouse: building a better model of multiple sclerosis
}

\author{
Richard M. Ransohoff \\ Neuroinflammation Research Center, Department of Neurosciences, Lerner Research Institute, Cleveland Clinic, Cleveland, Ohio, USA.
}

\begin{abstract}
The 2 cardinal cell populations mediating adaptive immunity are $\mathrm{T}$ and $\mathrm{B}$ lymphocytes. These cells play important but poorly understood roles in the immunopathological demyelinating disease multiple sclerosis (MS) and in a widely used animal model of human MS known as EAE. In the current issue of the JCI, 2 research teams report their parallel studies of double-transgenic mice expressing $T$ and $B$ cell receptors that recognize the same myelin protein (see the related articles beginning on pages 2385 and 2393). More than half of the double-transgenic mice spontaneously developed autoimmune demyelination in their spinal cords and optic nerves, exhibiting pathologies reminiscent of human MS. The studies describe an important new model for MS research.
\end{abstract}

MS is an acquired inflammatory demyelinating disorder of the human CNS, producing sensory, motor, and cognitive dysfunction in many affected individuals. For the past 75 years, much MS research has focused on EAE, an animal model of human MS. This useful model for scientific investigation (1) has been bedeviled by requirements for immunization, most often using myelin protein fragments along with powerful multicomponent adjuvants. The immunization protocols, especially the use of adjuvants, introduce complexity in data interpretation. Efforts to develop spontaneous EAE models have proceeded with ever-accelerating intensity in recent years. In the current issue of the JCI, 2 independent research groups report what I believe to be a new model of spontaneous autoimmune demyelination $(2,3)$. Given its simplicity and predictability, this model holds great promise as a system for studying MS pathogenesis. Both groups emphasize that lesion distribution in the so-named opticospinal EAE (OSE) mice resembles that seen in patients with Devic disease (also referred to as neuromyelitis optica [NMO]). However, recent advances in NMO research are leading toward a new

Nonstandard abbreviations used: AQP4, aquaporin4; BCR, B cell receptor; IgH ${ }^{\mathrm{MOG}}$ mice, MOG-specific Ig heavy-chain knock-in mice on a C57BL/6 background; MOG, myelin oligodendrocyte glycoprotein; NMO, neuromyelitis optica; OSE, opticospinal EAE; TCR ${ }^{\mathrm{MOG}}$ mice, MOG-specific TCR transgenic mice on a C57BL/6 background.

Conflict of interest: The author has declared that no conflict of interest exists.

Citation for this article: J. Clin. Invest. 116:2313-2316 (2006). doi:10.1172/JCI29834. nosology for human demyelinating diseases. Given this consideration and the differences in the neuropathology of murine OSE compared with human NMO, I suggest that this model will make its most salient contributions to MS rather than NMO research.

\section{Important and largely harmonious findings of the current studies}

Krishnamoorthy et al. (2) and Bettelli et al. (3) bred and studied essentially identical strains of double-transgenic mice, derived from 2 previously described singletransgenic animals $(4,5)$ (Figure 1$)$. The researchers performed overlapping analyses so that most findings (but not all) were instantly validated by independent evaluation. The first line of single-transgenic mice (4) was generated with knock-in technology, inserting the recombined heavy chain of a demyelinating anti-myelin oligodendrocyte glycoprotein (anti-MOG) antibody $(8.18 \mathrm{C} 5)$ in the Ig J region, yielding mice in which $20-30 \%$ of circulating B cells produced anti-MOG antibodies and expressed a $\mathrm{B}$ cell receptor (BCR) that recognized MOG protein. These MOG-specific Ig heavy chain knock-in mice on a C57BL/6 background were termed IgH ${ }^{\mathrm{MOG}}$ mice. The second line, termed TCR ${ }^{\mathrm{MOG}}$ mice, was generated by expressing a TCR that recognized an immunodominant determinant of MOG (5), and these mice spontaneously showed inflammation of the optic nerve, a typical early symptom of MS.

MOG is a stoichiometrically minor myelin protein but looms large in MS research: MOG immunization elicits autoimmune demyelination of varying types in rodents and nonhuman primates, with histological findings that recapitulate the gamut of MS pathology (6). MOG is expressed virtually exclusively in the CNS (but not in thymus), and MOG-reactive lymphocytes evade central thymic tolerance $(7,8)$. MOG is inserted into the external leaflet of the myelin membrane, so it is accessible to demyelinating antibodies, unlike other myelin components, which are buried deep within compact myelin $(9,10)$.

The 2 research groups reported exciting and concordant data $(2,3)$. First, approximately $50 \%$ of the double-transgenic $\mathrm{TCR}^{\mathrm{MOG}} \times \mathrm{IgH}^{\mathrm{MOG}}$ offspring spontaneously developed a severe form of EAE by 8 weeks of age and showed selective distribution of inflammatory lesions predominantly in their optic nerves and spinal cords, with cells in both meninges and parenchyma involved. The mouse model was descriptively termed OSE by Krishnamoorthy et al. (2). In the spinal cord, lesions were primarily subpial, as seen in active immunizationinduced EAE. By contrast, spontaneous EAE occurred in most previously reported models only at low incidence or following exposure to microbes or by eliminating regulatory cells (11-14). Both of the current reports found OSE severity and incidence unaffected by the usual strong influences such as gender and housing conditions $(2,3)$. Second, B cells in TCR ${ }^{\text {MOG }} \times I_{\text {IgH }}{ }^{M O G}$ mice presented antigen and also received $\mathrm{T}$ cell help, reflected by $\mathrm{B}$ cell proliferation and class switching to IgG1 (Figure 1). Crossing $\mathrm{IgH}^{\mathrm{MOG}}$ mice with transgenic animals bearing non-MOG-specific TCRs (i.e., OVA-specific TCR transgenic mice on C57BL/6 background) did not induce EAE (2), indicating that spontaneous OSE was not equivalent to the opening of the bloodbrain barrier to pathogenic antibodies by activated non-MOG-specific T cells (15). In vitro, activation of $\mathrm{B}$ cell proliferation was not dependent solely upon $T$ cells that recognized MOG (3). Amazingly, Krishnamoorthy et al. found that class switching from IgM to IgG1 MOG-specific antibod- 


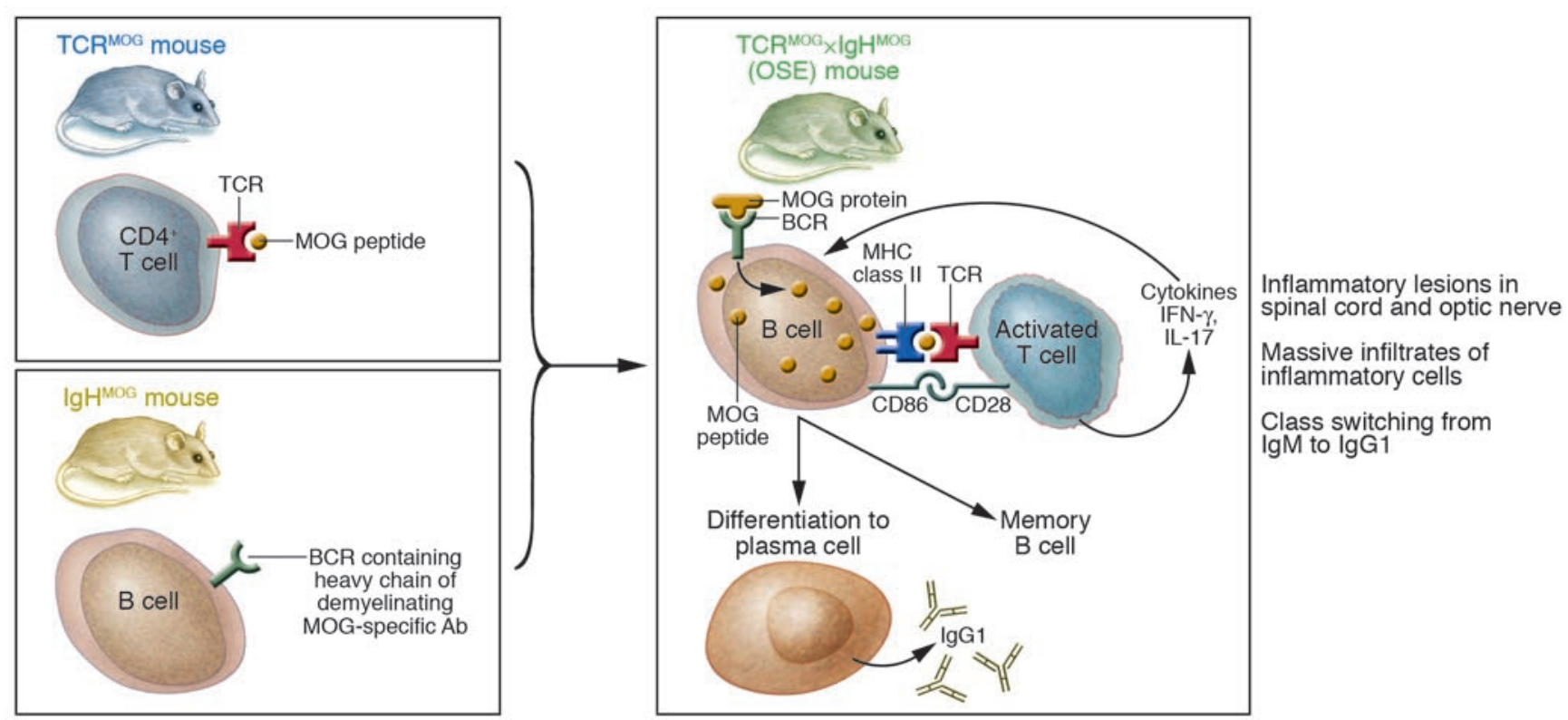

Figure 1

A mighty mouse for MS research. In this issue of the $\mathrm{JCl}$, 2 research groups developed double-transgenic mice (TCRMOG $\times$ IgHMOG mice, or OSE mice) expressing T and B cell receptors that recognize the same myelin protein, MOG $(2,3)$. More than $50 \%$ of these mice developed inflammatory demyelinating lesions in their spinal cords and optic nerves, reminiscent of human MS. The studies suggest that B cells play a critical role as APCs in OSE mice. B cells are able to present antigen efficiently because (a) the antigen receptor concentrates antigen from very low levels; (b) engagement of the antigen receptor generates signals that upregulate costimulatory molecules like CD86; and (c) antigen is shuttled to a compartment where it competes well for binding to $\mathrm{MHC}$ class II for presentation to $\mathrm{T}$ cells. Activated T cells then express the proinflammatory cytokines IFN- $\gamma$ and IL-17. MOG-specific B cells were also shown to undergo class switching to IgG1 in the presence of transgenic $T$ cells; however, this occurred in all double-transgenic mice whether they were asymptomatic or had clinical EAE, suggesting that anti-MOG IgG1 antibodies are not required to trigger disease.

ies occurred, even in $\mathrm{MOG}^{-/-}$mice, raising the question of whether a cross-reactive self antigen might be involved in the peripheral immune response that initiated spontaneous OSE. Third, cytokine production in OSE mice resembled that seen in active immunization-induced EAE in the same background mouse strain as the transgenic animals in the current studies. Cytokine profiles obtained from cell cultures and inflamed CNS tissues were similar and were characterized by a mixture of Th 1 and Th2 cytokines including IFN- $\gamma$, IL-5 (not typically detected in active immunizationinduced EAE in this mouse strain), and lesser levels of IL-17, a cytokine of great interest in contemporary EAE research.

\section{Immediate questions for a promising disease model}

OSE holds enormous potential as a model for MS. The OSE mouse is a robust and convenient spontaneous EAE model and will support significant mechanistic studies. So what are B cells doing in OSE? Findings from these 2 reports strongly implicate B cells as critical APCs in OSE. $B$ cells have marked advantages as APCs: antigen capture and internalization occurs at extraordinarily low antigen concentrations, mediated by the high-affinity B cell antigen receptor. Furthermore, signaling through the B cell antigen receptor following antigen engagement can promote antigen degradation to peptides and loading on MHC class II molecules (16) (Figure 1). The roles of B cells as APCs can be dissected in the OSE model. Current interest in anti-B cell therapeutics such as rituximab will be informed by this direction of research.

Another interesting question that remains to be answered concerns the abrupt onset of disease, which affords an opportunity to elucidate whether this pattern of disease evolution reflects the action of precipitating factors or the accumulation of pathogenic elements that exceed some threshold. As another example of research that may be enabled by this model, the seeming paradox concerning IFN- $\gamma$ as an inhibitory cytokine in EAE has been attributed to its interaction with adjuvants containing mycobacterial components (17); if so, IFN- $\gamma$ might function differently in OSE.

As noted above, Krishnamoorthy et al. (2) found evidence for IgH ${ }^{\text {MOG }}$ B cell class switching in $\mathrm{MOG}^{-/-}$mice that lacked nominal antigen, suggesting that there may be another ligand for the transgenic $\mathrm{IgH}^{\mathrm{MOG}}$ BCR and possibly for the TCRMOG TCR as well. Clarifying this fascinating finding might identify mechanisms by which crossreactivity to self antigens in the periphery leads to CNS autoimmunity.

It remains uncertain whether the mechanisms underlying the selective and provocative occurrence of OSE lesions in the optic nerve and spinal cord will be susceptible to elucidation. It seems unlikely that this lesion distribution is determined by regional abundance of MOG, as suggested by Bettelli et al. (3), given the vast variety of CNS demyelinating syndromes mediated by autoimmunity to MOG $(6,18-20)$. It will be important to interrogate the local vascular anatomy and genetics of the model for answers.

\section{Some discrepancies remain unresolved}

With regard to the presence of $B$ cells in the lesions, Bettelli et al. reported aggregates of B220-immunoreactive leukocytes (3). In contrast, Krishnamoorthy et al. did not 


\section{Table 1}

Few similarities and many differences between OSE mice and NMO patients

\author{
Characteristic \\ Pattern of neurobehavioral deficit evolution \\ Spinal lesion pathology
}

Evidence for humoral contribution to pathogenesis

Lesion location and extent

Presence of AQP4 antibodies

\section{OSE mice}

Abrupt, nonremitting

Subpial inflammation, demyelination; eosinophils present (2)

Burden of evidence against (no relation of $\mathrm{Ig}$ levels or presence to EAE severity; no detectable deposition of Ig or complement in tissues) Lesions restricted to 1 interspace No

\begin{abstract}
NMO patients
Abrupt, often nonremitting

Necrotizing inflammation with eosinophils; destruction of gray and white matter; hyalinized small vessels

Preponderance of evidence favors (readily detectable Ig and complement in tissues; clinical benefit with plasma exchange) Lesions extend for at least 2 interspaces Yes
\end{abstract}

Results shown from studies in this issue of the $J C /$ by Krishnamoorthy et al. (2) and Bettelli et al. (3).

detect $\mathrm{B}$ cells using immunohistochemistry in affected spinal cord and buttressed these negative findings by showing virtually no $\mathrm{CD}^{-} \mathrm{B} 220^{+}$cells in lysates of the inflamed CNS tissues (2). It is not likely that technical differences underlie this incongruity, as tissue analysis in both cases was performed by very experienced research neuropathologists. In addition, Krishnamoorthy et al. found a very impressive eosinophilic contribution to the leukocyte populations in lesions (2), while Bettelli et al. specifically reported the absence of eosinophils (3). These differences will need to be unraveled if the model is to bear utility for research, particularly for interventional studies to ameliorate or prevent disease.

\section{What features distinguish OSE mice from NMO patients?}

Despite the superficial resemblance conferred by lesion distribution of the phenotype of the double-transgenic mice to human NMO, the present reports describe a pathology that much more resembles MS or EAE than NMO. It is unfortunate that we do not have an animal model of NMO, a variant and relatively uncommon form of human demyelinating disease that has presented a fertile field for research. Recent work on NMO provides hope that studying variant forms of CNS demyelinating disease might allow researchers to recapitulate the remarkable progress made by investigators into the inflammatory peripheral nerve syndromes (21-23).

\section{Several key features distinguish human NMO pathology from that seen in OSE mice:}

Lesion pathology. Human NMO possesses a distinct pathology characterized by inflammatory infiltrates that include polymor- phonuclear leukocytes and eosinophils, hyalinized small-caliber spinal arteries, and necrotic destruction of both gray and white matter within the affected spinal cord in addition to lymphocyte and macrophage infiltrates (24-26). Deposition of complement C9 neoantigen and Ig bear testimony to the proposed humoral pathogenesis of NMO (26). In support of the concept of pathogenic humoral immunity in NMO, plasma exchange can be strikingly effective for the treatment of patients with severe attacks. Characteristics of NMO pathology that were specifically sought - but absent - in OSE mice included deposition of complement and Ig in the inflamed CNS as well as necrotic tissue damage and vascular hyalinization. Furthermore, there was no temporal relationship between Ig class switching and the presence or severity of EAE neurological deficits in OSE mice (2), arguing against a direct role for antibody in disease pathogenesis.

Lesion extent. Longitudinally extensive spinal lesions are reported to produce nonremitting paralysis in patients with $\mathrm{NMO}$ and constitute a major supporting diagnostic criterion (27). These longitudinally extensive lesions, typical of NMO, were absent from the OSE mice described in these studies. By contrast, spinal lesions in OSE animals were relatively small.

Aquaporin-4 antibodies. About 55\% of NMO patients harbor serum autoantibodies, initially characterized as NMO-Ig (28). Studies with NMO patients and appropriate controls, most importantly patients with classical MS and paraneoplastic syndromes, suggested that the detection of NMO-Ig was a specific indicator for the presence of disorders within the NMO spectrum (28). Further study identified an NMO-Ig antigen: aquaporin-4 (AQP4), a water channel highly expressed on the astrocytic end-feet around CNS vessels (29). This serological reactivity represents the first potential biomarker of a subtype of human demyelinating disease and opens the possibility of a molecular nosology for these complex disorders. It remains uncertain whether AQP4 antibodies are pathogenic in NMO, although their presence in patients with spinal cord demyelination predicts relapse (25). There was no reason to suspect that OSE mice should possess AQP4 antibodies, and they did not (2). For now, NMO is pathologically and, at a molecular level, etiologically distinct from OSE (Table 1).

\section{Summary}

In this issue of the JCI, 2 groups of leading scientists generated and characterized what I believe to be a new model of spontaneous EAE (2, 3). Already, their work provides new insight into the potency of $\mathrm{B}$ cells as APCs. There are tantalizing hints that MOG-specific antigen receptors can also "see" a component distinct from MOG in $\mathrm{MOG}^{-/-}$mice. It is anticipated that full exploitation of this spontaneous model will help resolve confusion that has arisen from the need to use immunization with adjuvants, as applied in most prior EAE studies. Prospects for new MS therapeutics are brighter due to the availability of this useful experimental system.

Address correspondence to: Richard M. Ransohoff, Neuroinflammation Research Center, Department of Neurosciences, Lerner Research Institute, Cleveland Clinic, Mail Code NC30, 9500 Euclid Avenue, Cleveland, Ohio 44195, USA. Phone: (216) 444-0627; Fax: (216) 444-7927; E-mail: ransohr@ccf.org. 
1. Ransohoff, R.M. 2006. EAE: pitfalls outweigh virtues of screening potential treatments for multiple sclerosis. Trends Immunol. 27:167-168.

2. Krishnamoorthy, G., Lassmann, H., Wekerle, H., and Holz, A. 2006. Spontaneous opticospinal encephalomyelitis in a double-transgenic mouse model of autoimmune $\mathrm{T}$ cell/B cell cooperation. J. Clin. Invest. 116:2385-2392. doi:10.1172/JCI28330.

3. Bettelli, E., Baeten, D., Jäger, A., Sobel, R.A., and Kuchroo, V.K. 2006. Myelin oligodendrocyte glycoprotein-specific $\mathrm{T}$ and $\mathrm{B}$ cells cooperate to induce a Devic-like disease in mice. J. Clin. Invest. 116:2393-2402. doi:10.1172/JCI28334.

4. Litzenburger, T., et al. 1998. B lymphocytes producing demyelinating autoantibodies: development and function in gene-targeted transgenic mice. J. Exp. Med. 188:169-180.

5. Bettelli, E., et al. 2003. Myelin oligodendrocyte glycoprotein-specific $\mathrm{T}$ cell receptor transgenic mice develop spontaneous optic neuritis. J. Exp. Med. 197:1073-1081.

6. Storch, M.K., et al. 1998. Autoimmunity to myelin oligodendrocyte glycoprotein in rats mimics the spectrum of multiple sclerosis pathology. Brain Pathol. 8:681-694.

7. Wekerle, H., and Linington, C. 2006. Organ specific autoantigens and the autoreactive $T$ cell repertoire: the case of myelin oligodendrocyte glycoprotein. Eur. J. Immunol. 36:512-515.

8. Fazilleau, N., et al. 2006. Persistence of autoreactive myelin oligodendrocyte glycoprotein (MOG)specific T cell repertoires in MOG-expressing mice. Eur. J. Immunol. 36:533-543.

9. Kroepfl, J.F., Viise, L.R., Charron, A.J., Linington, C., and Gardinier, M.V. 1996. Investigation of myelin/oligodendrocyte glycoprotein membrane topology. J. Neurochem. 67:2219-2222.

10. Mathey, E., Breithaupt, C., Schubart, A.S., and Linington, C. 2004. Commentary: Sorting the wheat from the chaff: identifying demyelinating components of the myelin oligodendrocyte glycoprotein (MOG)-specific autoantibody repertoire. Eur. J. Immunol. 34:2065-2071.

11. Madsen, L.S., et al. 1999. A humanized model for multiple sclerosis using HLA-DR2 and a human T-cell receptor. Nat. Genet. 23:343-347.

12. Goverman, J. 1999. Tolerance and autoimmunity in TCR transgenic mice specific for myelin basic protein. Immunol. Rev. 169:147-159.

13. Furtado, G.C., et al. 2001. Regulatory T cells in spontaneous autoimmune encephalomyelitis. Immunol. Rev. 182:122-134.

14. Waldner, H., Whitters, M.J., Sobel, R.A., Collins, M. and Kuchroo, V.K. 2000. Fulminant spontaneous autoimmunity of the central nervous system in mice transgenic for the myelin proteolipid proteinspecific T cell receptor. Proc. Natl. Acad. Sci. U. S. A. 97:3412-3417.

15. Westland, K.W. et al. 1999. Activated non-neura specific $\mathrm{T}$ cells open the blood-brain barrier to circulating antibodies. Brain. 122:1283-1291.

16. Rodriguez-Pinto, D. 2005. B cells as antigen presenting cells. Cell. Immunol. 238:67-75.

17. Matthys, P., Vermeire, K., Heremans, H., and Billiau, A. 2000. The protective effect of IFN-gamma in experimental autoimmune diseases: a central role of mycobacterial adjuvant-induced myelopoiesis. J. Leukoc. Biol. 68:447-454.

18. Kawakami, N., et al. 2004. The activation status of neuroantigen-specific $\mathrm{T}$ cells in the target organ determines the clinical outcome of autoimmune encephalomyelitis. J. Exp. Med. 199:185-197.
19. Linington, C., et al. 1993. T cells specific for the myelin oligodendrocyte glycoprotein mediate an unusual autoimmune inflammatory response in the central nervous system. Eur. J. Immunol. 23:1364-1372.

20. Berger, T., et al. 1997. Experimental autoimmune encephalomyelitis: the antigen specificity of $\mathrm{T}$ lymphocytes determines the topography of lesions in the central and peripheral nervous system. Lab. Invest. 76:355-364.

21. Stadelmann, C., and Bruck, W. 2004. Lessons from the neuropathology of atypical forms of multiple sclerosis. Neurol. Sci. 25(Suppl. 4):S319-S322.

22. Hughes, R.A., and Cornblath, D.R. 2005. GuillainBarre syndrome. Lancet. 366:1653-1666.

23. Willison, H.J. 2006. Basic and clinical aspects of autoimmune disorders in peripheral nerves. Acta Neurol. Scand. Suppl. 183:14-18.

24. Cree, B.A., Goodin, D.S., and Hauser, S.L. 2002. Neuromyelitis optica. Semin. Neurol. 22:105-122.

25. Giovannoni, G. 2006. Neuromyelitis optica and anti-aquaporin-4 antibodies: widening the clinical phenotype. J. Neurol. Neurosurg. Psychiatry. doi:10.1136/jnnp.2006.090944

26. Lucchinetti, C.F., et al. 2002. A role for humoral mechanisms in the pathogenesis of Devic's neuromyelitis optica. Brain. 125:1450-1461.

27. Wingerchuk, D.M. 2006. Neuromyelitis optica. Int. MS J. 13:42-50.

28. Lennon, V.A., et al. 2004. A serum autoantibody marker of neuromyelitis optica: distinction from multiple sclerosis. Lancet. 364:2106-2112.

29. Lennon, V.A., Kryzer, T.J., Pittock, S.J., Verkman, A.S., and Hinson, S.R. 2005. IgG marker of opticspinal multiple sclerosis binds to the aquaporin-4 water channel. J. Exp. Med. 202:473-477.

\title{
Regeneration of the endothelium as a novel therapeutic strategy for acute lung injury
}

\author{
Tohru Minamino and Issei Komuro
}

Department of Cardiovascular Science and Medicine, Chiba University Graduate School of Medicine, Chiba, Japan.

\begin{abstract}
Acute lung injury (ALI) is characterized by the influx of protein-rich edematous fluid into the airspaces due to increased permeability of the alveolarcapillary barrier. Inflammatory mediators are thought to play a critical role in the pathogenesis of this disorder. In this issue of the JCI, Zhao et al. report that the forkhead box M1 (FoxM1) transcription factor induces endothelial regeneration and thereby restores endothelial barrier function after ALI (see the related article beginning on page 2333). Their findings raise the intriguing possibility that the promotion of endothelial regeneration may be a novel therapeutic strategy for ALI.
\end{abstract}

\section{Pathophysiology of acute lung injury}

Acute lung injury (ALI) and its more severe form, acute respiratory distress syndrome

Nonstandard abbreviations used: ALI, acute lung injury; ARDS, acute respiratory distress syndrome; FoxM1, forkhead box M1; FoxM1 CKO, endothelial cell-restricted FoxM1-deficient (mice).

Conflict of interest: The authors have declared that no conflict of interest exists.

Citation for this article: $J$. Clin. Invest. 116:2316-2319 (2006). doi:10.1172/JCI29637.
(ARDS), are characterized by an acute inflammatory process in the airspaces and lung parenchyma (1). These clinical syndromes are manifestations of the loss of barrier function of the alveolar epithelial and pulmonary capillary endothelial cells, resulting in respiratory failure. Epidemiological data suggest that the annual incidence of ALI/ARDS in the United States is 75 per 100,000 of the population. Although evidence exists that mortality in patients with ALI/ARDS has declined over the last 10 years, it remains high at $30 \%-40 \%$ and is still an important cause of death in critically ill patients.

The clinical course of ALI is a complex, variable process associated with severe lung dysfunction. The first stage, the exudative phase, is an acute inflammatory response accompanied by a marked influx of neutrophils injuring epithelial and endothelial cells (Figure 1). The resulting death of type I epithelial cells invites a breakdown in the gas exchange and barrier function of the lung and is associated with the flooding of airspaces with protein-rich edematous fluid. Histological features are dense hyaline membranes and alveolar collapse. Injury to type II epithelial cells reduces surfactant production and impairs the removal of edematous fluid from the alveolar space. Endothelial injury leads to a widening of 